\title{
La innovación: baluarte fundamental para las organizaciones
}

\section{Innovation: fundamental bulwark for organizations}

\author{
Gabriel Diaz Muñoz \\ David Guambi Espinosa \\ Universidad Tecnológica Equinoccial, Ecuador
}

Autor para correspondencia: gabriel.diaz@ute.edu.ec, david.guambi@ute.edu.ec

Fecha de recepción: 21 de septiembre de 2018 - Fecha de aceptación: 15 octubre de 2018

Resumen: La innovación constituye el motor impulsor del desarrollo y crecimiento empresarial hacia la competitividad en un mercado cada vez más exigente y demandante. El presente documento persigue la finalidad de analizar la innovación desde diferentes perspectivas entendiéndola como una herramienta estratégica que coadyuva al cambio, diferenciación y permanencia en el mercado. La metodología utilizada para recopilar la información contenida en este documento se ha basado en un estudio bibliográfico y de carácter descriptivo, correlacional y cualitativo cimentado en la teoría y perspectivas que diferentes autores tienen acerca de la temática propuesta. Cada uno de ellos ha realizado aportes importantes que ayudan a comprender con facilidad la realidad de las empresas y la importancia que tiene la incorporación de procesos y estrategias direccionadas y enfocadas en la innovación. Este trabajo tiene como fin único facilitar la comprensión del tema en cuestión valiéndose para tal efecto de un análisis detallado, coherente, propositivo y cuestionable, pues lo interesante de relacionar las teorías de un autor con las de otro es precisamente la divergencia de criterios y puntos de vista que cada uno tiene, por cuanto coincidir en el interés por conocer sobre algún tópico en particular no implica necesariamente concordar en los razonamientos, visión o pensamientos ideológicos

Palabras Clave: innovación; empresa; competitividad; crecimiento empresarial; conocimiento Abstract: Innovation is the driving force of business development and growth towards competitiveness in an increasingly demanding and demanding market. This document aims to analyze innovation from different perspectives, understanding it as a strategic tool that contributes to change, differentiation and permanence in the market. The methodology used to compile the information contained in this document has been based on a descriptive, correlational and qualitative bibliographical study based on the theory and perspectives that different authors have about the proposed topic. Each of them has made important contributions that help to easily understand the reality of companies and the importance of incorporating processes and strategies directed and focused on innovation. This work has as its sole purpose, to facilitate the understanding of the subject in question, making use of a detailed, coherent, proactive and questionable analysis, because the interesting thing of relating the theories of one author with those of another is precisely the divergence of criteria and points of view that each one has, as to coincide in the interest to know about a particular topic does not necessarily imply to agree on the reasoning, vision or thoughts.

Key Words: innovation; company; competitiveness; business growth; knowledge 


\section{Introducción}

Reinventar ha sido la forma en que muchas organizaciones han podido dejar en el pasado viejos paradigmas que nos les permitían alcanzar el éxito, pues la innovación facilita a las empresas interactuar de forma dinámica y competitiva, y conseguir una participación importante en el mercado. La economía del conocimiento, los requerimientos de consumidores cada vez más exigentes, la evolución de la ciencia y la tecnología, la competitividad y la rivalidad entre las empresas son solo algunos factores que obligan a las organizaciones a hacer de la innovación no una opción sino una necesidad. El producto o servicio original no puede ser innovador por siempre o pensar que no requiere cambios ni mejoras argumentando que las ventas se mantienen estables.

El mundo de los negocios ha dado un giro de 180 grados y, para sobrevivir a la exigencia de los mercados, las empresas deben crear un entorno laboral direccionado a la innovación y mejora de los productos o servicios como factor de supervivencia. En el entorno empresarial que envuelve a las organizaciones, los cambios se producen con mayor rapidez con base en la demanda y requerimientos del mercado. Ante tal situación, las organizaciones deben tener no solo capacidad de adaptación a dichos cambios sino contar con los recursos humanos y económicos a fin de evolucionar con el pasar de los años.

La innovación implica riesgos, por ende, no está concebida para todas las empresas en su totalidad, pero aquellas que consiguen aplicarla, no solo que logran alcanzar la competitividad y crecen económicamente, sino que dan a sus productos o servicios características únicas y diferentes difíciles de imitar por la competencia. Sin embargo, eso no es todo: la innovación debe ir acompañada de una fuerte campaña de marketing a fin de dar a conocer la propuesta al mercado y que este lo acoja con agrado, caso contrario será un desperdicio innecesario de tiempo, recursos humanos y económicos.

Según Maqueda (2010), no vale únicamente con inventar un producto o servicio nuevo, lo que genera valor es introducirlo adecuadamente en el mercado y que este lo acepte.

\section{Desarrollo}

La innovación comprende un tópico de interés y constituye un baluarte indispensable para las organizaciones, toda vez que estas tengan como meta alcanzar la competitividad como factor de desarrollo y crecimiento sostenido a través del tiempo. Con el paso de los años, diferentes autores han emitido conceptos y teorías acerca de la innovación empresarial y algunos elementos vinculados a ella, los cuales se abordarán en los párrafos posteriores a fin de facilitar la comprensión del lector.

\section{La innovación}

"Hoy en día la innovación es un proceso clave de las empresas pues permite la creación de ventajas competitivas gracias a la introducción de productos y servicios nuevos o mejorados al mercado, y respalda su eficiencia productiva y organizacional gracias a la introducción o mejora de los procesos de producción y entrega". (Medellín, 2013, pág. 21). 
Actualmente, ya no es suficiente crear productos u ofrecer un servicio de calidad; es importante también el desarrollo de estrategias que, en mediano y largo plazo, se traduzcan en una ventaja competitiva difícil de copiar o imitar por la competencia a fin de hacer de ella una fortaleza que permita alcanzar los objetivos organizacionales.

"La innovación es un concepto extenso que comprende una amplia gama de actividades y procesos: mercados, actividades empresariales, redes y competencia, pero también las habilidades y organizaciones, la creatividad y la transferencia de conocimientos" (OCDE, 2013, pág. 17).

Joseph Schumpeter definió en 1934 la innovación como la introducción en el mercado de un nuevo producto o proceso, capaz de aportar algún elemento diferenciador, la apertura de un nuevo mercado o el descubrimiento de una nueva fuente de materias primas o productos intermedios.

En la medida que han ido transcurriendo los años, este concepto ha ido moldeándose hasta llegar a la actual definición propuesta en el Manual de Oslo (2005), en el que se advierte que la innovación es la introducción de un nuevo, o significativamente mejorado, producto o servicio, de un proceso, de un nuevo método de comercialización o de un nuevo método organizativo en las prácticas internas de la empresa.

No obstante, Peter Drucker relaciona la innovación como una provisión de más y mejores bienes y servicios, pero que no es suficiente para el negocio proveer cualquier bien y servicio, deben proveerlos mejores y más económicos. Las condiciones del entorno empresarial engloban un punto importante que no puede pasar desapercibido; de ellas dependerán que la estrategia propuesta por la cúpula organizacional promueva o frene la innovación, pues esta es, en un sentido más amplio, el reto de los directivos empresariales por plasmar la teoría en acciones generando ideas creativas que, a su vez, se puedan traducir en productos y servicios mejorados e insertados exitosamente en el mercado.

Corma (2013) señala que se entiende por innovación la conversión de ideas y conocimiento en productos, procesos o servicios mejorados para el mercado, para satisfacer así las necesidades de los ciudadanos, empresas y administraciones públicas.

El desafío de transmitir con eficiencia la estrategia plateada por la gerencia a todos los stakeholders en sus diferentes niveles no siempre puede cumplirse. Con frecuencia, las organizaciones son incapaces de establecer mecanismos que permitan incorporar actividades fiables y eficaces enfocadas en el crecimiento empresarial y desarrollo de nuevos productos o servicios de modo que se asegure la innovación como un proceso de naturaleza cíclica.

Surgen así, a partir de tal situación, cuestionamientos como: ¿Son realmente las empresas que han desarrollado mecanismos de innovación constante más eficientes que aquellas carentes de estos? o ¿Es necesario que los trabajadores en la empresa aporten con ideas para crear nuevos productos y servicios o esta tarea es exclusiva de la gerencia? Estas son sin duda preguntas que se pretenden responder al culminar este documento, luego de analizar una serie de elementos que envuelven al amplio e interesante concepto de innovación. 
En ocasiones, resulta complicado pero indispensable que las empresas evalúen periódicamente aquellos elementos que les afecta en su crecimiento económico a fin de tomar medidas correctivas en busca de mejorar y diseñar un plan de acción que permita superar esos obstáculos. La innovación, tan valorada en el sector empresarial, no puede llevarse a cabo por sí sola, necesita estar ligada a otros factores importantes como la investigación y el conocimiento, pues estos constituyen los elementos diferenciadores entre empresas que realizan sus procesos con monotonía y aquellas que se preocupan por buscar una manera distinta y más atractiva de ofrecer sus productos o servicios a sus clientes.

Rothwell (1992) define la innovación como un proceso que incluye la técnica, el diseño, la fabricación y las actividades comerciales y de gestión implicadas en la venta de un nuevo producto o el uso de un nuevo proceso de fabricación o equipamiento.

Es por ello que la necesidad de innovación debe ir direccionada no solo al producto o a los activos fijos tangibles sino al cúmulo de elementos a partir de los cuales la organización realiza sus procesos productivos de manera que los stakeholders perciban el ambiente laboral como una oportunidad para desarrollar su creatividad y aportar al cambio y mejora continua.

Autores como García (2009) sostienen que una empresa innovadora es aquella que, consciente de los cambios en el entorno, es capaz de introducir mejoras a sus productos o en el diseño e implementación de nuevos bienes y servicios, de manera que todo ello redunde en la aparición de ventajas competitivas para la empresa.

La demanda y requerimientos del mercado obligan a las organizaciones a pensar de manera diferente. Muchas veces se cree equivocadamente que, por el hecho de usar la frase "estamos innovando", el cliente llegará como por arte de magia a la empresa, pero la realidad es distinta: el consumidor aspira a recibir algo único y diferente por parte de la industria y es precisamente lo que se pretende lograr por medio de la innovación.

\section{La incertidumbre de innovar}

"La esencia de la innovación está en la gestión de la incertidumbre y de las oportunidades frente a la gestión de los recursos clásica de los administradores” (Arboníes, 2013, pág. 14).

La innovación comprende un desafío que actualmente buscan todas las empresas; no es algo sencillo pero con la estrategia correcta, la iniciativa y el liderazgo proveniente de la cúpula empresarial, la balanza se inclinará más en favor del éxito que del fracaso. Es común sentir cierto grado de temor ante la decisión de implementar procesos no antes realizados en la industria, la incertidumbre abraza cuando existe la iniciativa de salir de la zona de confort y dejar de lado la rutina con la que se acostumbra a operar.

El tratar de buscar ideas, que para otros puedan no tener valor alguno o sentido, es lo que conduce a encontrar respuestas diferentes; pues si los procesos son los mismos que se vienen manejando algún tiempo atrás, no puede esperarse resultados diferentes.

Según Domingo (2013), la innovación por definición supone riesgo e incertidumbre, pero siguiendo una serie de principios básicos y de metodología se pueden evitar errores. 
La innovación por naturaleza supone cambio y todo proceso de cambio genera resistencia, pues es un hecho que esta actitud forma parte de la naturaleza del ser humano; no obstante, los cambios son muchas veces absolutamente necesarios y esenciales, de otra manera la sociedad en general se quedaría estancada y paralizada sin avances de ninguna índole en el plano científico, tecnológico, cultural y social. Para las organizaciones, es indispensable mantener una participación activa en el mercado. Constantemente, buscan la manera de ofrecer algo mejor y diferente; esto implica necesariamente el riesgo y la incertidumbre generados por el afán de producir cosas que los demás no están haciendo, dejando así abierta la posibilidad que el producto entregado al mercado tal vez no tenga la acogida y aceptación con la que se planificó en su fabricación.

Autores como Domingo (2013) sostienen que la innovación es el viaje a lo desconocido y, como tal, no se puede predecir lo que va a pasar; sin embargo, se puede conseguir que la probabilidad de éxito se incremente de forma dramática sistematizando el proceso y siguiendo los procesos y la metodología adecuados.

Cuando las organizaciones tienen la convicción de explorar terrenos desconocidos, el riesgo de equivocarse permanece latente y ligado a él la posibilidad de perder dinero. Es de ahí que surgen las inseguridades y el deseo de indagar si alguien más ya probó y le fue bien; pero, al ejecutar estas acciones, el resultado no llegará más allá de hacer lo que otros ya habían hecho con anterioridad.

Cuando la estrategia empresarial está direccionada a lograr solo ligeras mejoras, el riesgo y la incertidumbre tienden a disminuir; es decir, la organización seguirá mejorando en el día a día lo cual tiene una connotación positiva para el éxito y el crecimiento empresarial; eso no implica innovar y, como posiblemente la competencia utilice la misma lógica de disminuir el riesgo por temor a perder lo que hasta el momento ha logrado construir, el resultado de los productos o servicios entregados al consumidor no será necesariamente igual o parecido, pero quizás tenga características valoradas con cierta similitud por los clientes y esto se traduzca en el fracaso del intento por sobresalir y lograr diferenciación.

"Una empresa que haga las mismas cosas de la misma forma que la competencia no puede ser mejor que sus rivales y la competencia frontal con la que se enfrentará solo le garantizan con una parte muy pequeña del valor que cree” (Roberts, 2006, Pág. 212).

\section{Tipos de innovación}

Es común vincular a la innovación como una actividad de naturaleza creativa, donde la inspiración del momento da como resultado la creación o la mejora de un producto o servicio.

Para que se lleve a cabo el proceso de innovación, algunos componentes deben conjugarse a fin de generar ideas que puedan plasmarse en bienes con éxito comercial en el mercado.

El entorno que envuelve a las organizaciones es de naturaleza cambiante en temas sociales, tecnológicos y culturales. Las organizaciones no son ajenas a estos cambios debido a la interacción constante entre sus grupos de interés como clientes, competidores y proveedores, lo 
cual empuja y obliga a los directivos a considerar una estrategia empresarial en función de ellos. Cuando la empresa toma la decisión de innovar, es necesario conocer algunos factores que ayuden a comprender de mejor manera los gustos y preferencias del mercado, a identificar aquellos productos o actividades que puedan someterse al proceso de innovación y, sobre todo, a determinar si la industria tiene los recursos tecnológicos y económicos que impliquen la innovación con sus correspondientes costes y beneficios que se pretenden obtener al finalizar el proceso. Todas las áreas o departamentos que forman parte de la organización son susceptibles de incluirse en el proceso de innovación, de ahí se deriva la importancia de identificar aquellas que requieran una intervención inmediata y, a su vez, presten condiciones favorables para tal acción.

"Las innovaciones están presentes en cualquier área de la empresa, abarcan un amplio espectro de actividades que van desde las cotidianas hasta las estratégicas". (Martínez, López, García y Estrada, 2009, pág. 167).

Al hablar de innovación, no se puede limitar el concepto a la incorporación de ligeras mejorías en los procesos o en la inversión en tecnología y equipos: la innovación abarca más ampliamente todo el proceso de gestión empresarial de manera que se pueda ofrecer nuevas y atractivas soluciones a problemáticas u oportunidades identificadas que nadie ofrezca todavía o que el mercado espera con ansias pero aún no dispone. Muchas veces se asocia el término innovación a la creación de un nuevo producto o servicio, entendiendo que la industria requiere realizar grandes inversiones en maquinaria, tecnología, infraestructura y capacitación del personal; sin embargo, la innovación hace referencia también a la reestructuración de procesos y cambios que se generan en la organización mediante la implementación de actividades direccionadas a realizar las cosas de manera inusual o diferente a la acostumbrada sin que exista de por medio una inversión significativa para poder llevar a cabo este proceso.

Según Verde (2013), en su libro Soñar es poder, ser innovador no se refiere solo a crear algo que no existe sino también a ser más efectivo y crear mucho más con lo que se tiene a mano.

A lo largo de la historia, las empresas han incluido actividades innovadoras como herramientas estratégicas para ganar liderazgo en el mercado y. ligado a ello, ha habido una mejora notable en la calidad de vida de la población. Toda innovación tiene mayor o menor impacto en los consumidores y en el mercado en general; esto depende en gran medida de la magnitud del cambio y esto, a su vez, marca una diferencia entre empresas que tratan de innovar y lo único que consiguen son implementar ligeras mejoras pero fracasan en el intento por lograr algo exclusivo, mientras otras tantas que progresan generan mayores ingresos que la competencia por medio de actividades innovadoras, productos y servicios únicos y llegan a posicionarse en lugar de permanecer estancadas por muchos años.

Dussauge, Hart y Ramanantsoa (1992) señalan que las innovaciones pueden ser clasificadas según la magnitud del cambio que conllevan a partir de considerar los productos como sistemas que articulan componentes y conceptos. Se tiene la siguiente tipología: 
- Incremental: La articulación entre conceptos y componentes o arquitectura del producto no se cambia, únicamente se refuerzan o mejoran algunos de sus componentes o conceptos.

- Radical: Tanto la arquitectura como los componentes son alterados, de hecho se trata de un nuevo producto.

- Modular: Se cambian radicalmente los componentes modulares de un producto pero su arquitectura permanece sin cambio; el cambio de teléfonos analógicos a digitales es un ejemplo de este tipo de innovación tecnológica.

- Arquitectural: Se modifica la forma en que se articulan los componentes y conceptos del producto pero los componentes y conceptos únicamente se refuerzan o permanecen sin cambio, ejemplos de estos cambios se dan en los ordenadores personales y no son fácilmente identificables por los consumidores porque son a nivel sistema. (pág. 14 y 15)

Por otro lado, el Manual de Oslo (OCDE, 2005) define cuatro tipos de innovación que incluyen cambios sustanciales en las actividades de las empresas:

- Innovación de producto/servicio: Implican los cambios significativos de las características de los bienes o servicios; incluyen ambos, los bienes y los servicios enteramente nuevos y las mejoras significativas de los productos existentes.

- Innovación de proceso: Son cambios significativos en los procesos de producción, logística o distribución.

- Innovación organizacional: se refieren a la puesta en práctica de nuevos métodos de organización. Estos pueden ser cambios en las prácticas de la empresa, en la organización del lugar de trabajo o en las relaciones exteriores de la empresa.

- Innovación de mercadotecnia: Implican la puesta en prácticas de nuevos métodos de comercialización; estos pueden incluir cambios en el diseño y el envasado de los productos, en la promoción y la colocación de los productos y en los métodos de tarificación de los bienes y servicios. (pág 23 y 24)

\section{La innovación como herramienta estratégica hacia la competitividad}

Arboníes (2017) señala que la empresa es un ente vivo donde hacer y ser son inseparables. En algunos casos y momentos, genera impresionantes espirales virtuosas de desarrollo y competitividad; en otros, desarrollan proyectos poco atractivos que conducen a círculos viciosos o inclusive al fracaso.

Las condiciones del ambiente empresarial, en la actualidad, vislumbran que un futuro demandante y complejo se aproxima; las expectativas del mercado son más exigentes y la competencia entre las empresas se vuelve cada vez más difícil pues cada una trata de sacar su mejores armas de batalla para acaparar mayor clientela. Por tanto, la innovación se convierte en una herramienta estratégica imprescindible que todas las organizaciones deben tener para alcanzar niveles de competitividad aceptables y garantizar de esa manera la supervivencia.

Verde (2013) asegura que los emprendedores y las empresas están obligados a ser cada vez más competitivos y la única forma de conseguirlo es mediante la innovación constante.

Durante muchos años, se pensó equivocadamente que la innovación era una característica de superioridad que podía ser aplicada únicamente en determinadas ramas de la ciencia como la ingeniería y la medicina, o se adjudicaban exclusivamente las grandes empresas y, por ende, solo 
aquellas que cumplían con esta característica lograban ser competitivas, brindar servicios de calidad y entregar al mercado productos que cumplían e inclusive superaban las expectativas de los consumidores.

Autores como Muñoz (2014) sostienen que la innovación es parte de la naturaleza humana, es el deseo natural de crear algo nuevo e inventar un mejor futuro, pero hubo un tiempo en el que el proceso de creación estuvo monopolizado por magos, académicos, científicos y expertos.

Hoy se conoce en un sentido más amplio que cualquier empresa puede innovar, siempre y cuando el entorno laboral preste las condiciones favorables para liberar el potencial de los stakeholders y la cúpula empresarial tenga una visión clara de las áreas, productos o servicios que son susceptibles de incluirse en este proceso. Las condiciones económicas y las estructuras del mercado no son siempre las mismas; por ende, una empresa que lidera el mercado actualmente no es garantía de que pueda seguir haciéndolo en un futuro, los procesos pueden volverse obsoletos o simplemente el producto que entregaban al mercado ya no tiene la acogida y aceptación que tenía en un principio. Es por eso que pensar en la innovación como una herramienta generadora de valor agregado representa un verdadero reto para todas las organizaciones en la actualidad y para sus directivos, pues claro está que, para sobresalir y asegurar la continuidad de la empresa a través del tiempo, es necesario ser mejores que el resto.

Para Valdivia (2015), los clientes no solo buscan un buen producto o servicio sino que cada vez prestan más atención a los componentes o atributos intangibles; en este sentido, la estrategia comercial supone el desarrollo de una ventaja competitiva difícil de imitar por las empresas rivales.

Muchas empresas adjudican la falta de capacidad para innovar al limitado presupuesto o carencia de recursos económicos, lo cual supone que merma de manera importante el crecimiento en ventas, retener a la clientela actual y direccionarse hacia mercados distintos a la región donde operan. Es por eso que la exploración y estudio de nuevos mercados son elementos importantes que deben tenerse en cuenta como factores de crecimiento y supervivencia en tiempos de demanda baja o cuando el mercado local decae.

Cordero, Chavarría, Echeverri y Sepúlveda (2003) señalan que la competitividad se define como la capacidad de mantener y ampliar la participación de las empresas en los mercados locales e internacionales de una manera lucrativa que permita su crecimiento.

Es conocido por todos que las exigencias de los mercados, en general, han crecido con el pasar de los años de forma sorprendente; por tanto, se vuelve imprescindible manejar cuidadosamente todos los procesos productivos a fin de tener, como entregables, bienes o servicios que cumplan con las expectativas del consumidor y poder, así, destacar entre la diversidad de competidores.

\section{Los stakeholders como factor determinante}

La innovación empresarial no necesariamente se determina con base en la cantidad de recursos humanos que se destinan a este proceso sino más bien por la cultura innovadora con la 
que cada industria crea y desarrolla sus productos o servicios. En este contexto, el papel de los stakeholders en un elemento determinante para que todas las actividades y procesos se desarrollen en un entorno organizacional de confianza donde la creatividad y la innovación continua se manejen de forma permanente.

Si bien es cierto, es probable que las empresas tomen la decisión de incorporar procesos de innovación dentro de sus actividades, pero muchas veces los intentos por hacer las cosas de manera diferente a la acostumbrada solo terminan siendo un fracaso con los respectivos costes que representan; esto se genera en repetidas ocasiones por causa de un direccionamiento equivocado que prioriza invertir grandes cantidades de recursos económicos descuidando la importancia que debe tener, por un lado, fomentar un ambiente laboral con las condiciones necesarias para que se pueda llevar a cabo la innovación y, por otro, plantear la estrategia correcta que incluya al talento humano como eje central del proceso y esto redunde en el desarrollo personal y profesional de los stakeholders.

"Las empresas innovadoras, o que pretendan serlo, deben diseñar estrategias adecuadas para atraer el talento a su organización y cuidar y hacer crecer el existente" (García, 2009, pág. 82).

La sociedad actual, caracterizada por ser una época de continuos cambios y evolución hacia un universo digital, ha convertido a los stakeholders en un recurso esencial para las organizaciones que pueden aportar a la competitividad de la industria y su permanencia a través del tiempo por medio de ideas innovadoras que agreguen valor a los productos o servicios que la empresa entrega al mercado. Generalmente, la cultura organizacional, que predomina en la mayoría de las empresas, es aquella en la que la gerencia es la encargada de encontrar soluciones a las problemáticas que en algún momento la industria atraviesa; sin embargo, bajo este régimen solo se fomenta un sistema de gestión centralizado en las actividades y procesos, característica que era propia de las empresas del siglo pasado donde la estructura organizacional jerarquizada que predominaba terminaba anulando la posibilidad de que surjan nuevas ideas desde un punto de vista distinto al de los jefes.

El primer paso para que el entorno laboral sea propicio y afín a las actividades y procesos innovadores en la empresa es fomentar un ambiente de confianza en el que los stakeholders puedan expresar sus ideas y creencias sin el temor de ser criticados o censurados. Resulta de mucha ayuda también apoyarse en actividades en las que haya una constante interacción entre el talento humano y la cúpula organizacional, de manera que existan propuestas para resolver determinada situación escuchando diferentes opiniones, y esta acción sea vista como una inversión en busca de soluciones innovadoras mas no como una pérdida de tiempo.

Según Rodríguez (2017), los empleados son los principales promotores de ideas, sobre todo, para mejorar los procesos productivos. En muchos casos, se cambia el método, el orden o distribución de las fases de producción, lo que ahorra tiempo gracias a la innovación de los empleados.

Claro está que la gerencia es la encargada de marcar el camino a seguir y liderar el cambio de procesos rutinarios y monótonos hacia aquellos de naturaleza innovadora en la 
empresa; pero para que este cambio pueda darse, es necesario contar con talento humano que confíe en su propio trabajo y apueste por un pensamiento colaborativo, creativo e innovador. Es por ello que la propuesta de nuevas ideas o planteamiento de mejoras en los procesos productivos de la industria por parte de los stakeholders resulta un factor de desarrollo y crecimiento importante para todas las organizaciones en la actualidad.

Bermejo y López (2014), en su libro La innovación continua en el éxito empresarial, señalan que las empresas deben utilizar procesos de innovación abierta para capturar el máximo de ideas, asegurar que su personal disponga de tiempo para pensar en nuevos productos y servicios, y estar constantemente vigilando e interpretando las tendencias del mercado que puedan ser fuente de nuevas ideas.

\section{La cúpula empresarial y su liderazgo en el proceso de innovación}

Según Drucker (2014), el trabajo, para producir resultados, debe ser planificado y realizado con dirección, método y propósito.

Hoy en día, las organizaciones coinciden en la importancia que tiene el liderazgo como herramienta facilitadora del cambio e innovación en la industria. A los largo de los años, el liderazgo ha sido entendido como la capacidad de influir en el comportamiento de otras personas de manera que se promuevan los esfuerzos individuales y colectivos en pro de la consecución de los objetivos organizacionales; pero, más allá de una definición, es necesario comprender que el liderazgo se lleva a cabo mediante la materialización del actor principal en este proceso: el líder.

Desde tiempos milenarios, siempre ha existido la necesidad de contar con una persona capaz de asumir la responsabilidad de un grupo, velar por los intereses de otros y preocuparse por el bienestar común de un colectivo, puesto que la carencia de un individuo que cumpla con estas características deriva en la práctica de comportamientos improductivos y anárquicos que desembocan en el caos y la fragmentación de la organización. Es visible que el contexto económico actual de las empresas se vuelve cada vez más amplio e impredecible. Mantenerse en el mercado y retener la clientela actual resultan tareas complejas que requieren gerentes con visión, capacidad de dirección y sobre todo habilidades para desarrollar y potenciar el talento humano existente.

Autores como Martínez (2006) sostienen que las empresas actualmente se enfrentan a la necesidad de desarrollar capital humano, sistemas de información que gestionen de manera más efectiva la información y capacidades tecnológicas que les permitan seguir compitiendo en mercados altamente competitivos como los actuales.

Los competidores muestran agresividad y los consumidores a su vez son más exigentes, el entorno es cambiante y todo ello hace que la estabilidad económica de las organizaciones sea susceptible de cambio. Es por esto que los estilos de liderazgo y dirección tradicional han dado un giro de 180 grados en relación con lo que se manejaba años atrás. Por ende, aplicar modelos de gestión empresarial adecuados a las demandas y requerimientos de los tiempos actuales resulta indispensable para la industria. "El liderazgo es un fenómeno sumamente complejo que depende, no solo de la persona que eventualmente lo ejerce, sino también de los seguidores y de 
las circunstancias de la situación, que pueden demandarlo o no" (Castillo y Cubeiro, 2016, pág. 16).

Ciertamente, la innovación y el liderazgo son dos conceptos estrechamente ligados. Innovar requiere que un grupo de personas trabajen de forma cooperativa en un entorno donde la confianza y la fluidez comunicacional entre los diferentes departamentos permitan hacer de la innovación un ente generador de valor agregado y promotor de soluciones efectivas que aportan significativamente a la organización. Por otro lado, cuanto más alta es la posición del líder en la empresa, mayor relevancia tiene crear entornos que favorezcan la implementación de actividades y procesos innovadores que tienen como objetivo la transformación organizacional sostenible en mediano y largo plazo, es por esto que, el líder debe tener como objetivo principal ser el promotor de entornos y culturas innovadoras antes que tomar la iniciativa o actuar por sí mismo como innovador.

\section{La creatividad e innovación como factor de supervivencia para las organizaciones}

Actualmente, se reconoce la importancia que la creatividad e innovación tienen en la industria como fuentes de sustento y herramientas generadoras de valor a los productos o servicios que la empresa fabrica. Muchos entendidos en la materia podrían generar controversias ante la afirmación de que los líderes verdaderos pueden valerse de sus habilidades y capacidad de motivación al personal para promover la creatividad argumentando que la gente creativa, al igual que los buenos deportistas, nace más no se hace. En efecto, posiblemente la creatividad es un don otorgado a pocos; sin embargo, cabe señalar que todas las personas sin excepción alguna tienen aptitudes creativas que pueden incrementarse siempre y cuando el entorno preste las condiciones favorables para su florecimiento además de un correcto coaching y capacitación.

Perkins (1981), en su libro The Mind's Best Work, señala experimentos en los que se solicitó a diferentes personas, que formaban parte de un estudio, que piensen en voz alta o reporten sus pensamientos durante un episodio de invención. Las respuestas obtenidas indujeron a que Perkins concluya este estudio señalando que la creatividad surge naturalmente y de forma comprensible ante ciertas aptitudes cotidianas de percepción, entendimiento, lógica, memoria y estilo de pensar.

De este estudio, se deriva la importancia de que los líderes estimulen la creatividad con el fin de evitar la obsolescencia de los productos, bienes o servicios que comercializa la industria por un lado y que, mediante la creación e innovación de procesos productivos y los bienes que se obtienen a partir de ellos, se pueda incrementar la productividad de la empresa y asegurar su permanencia en el tiempo. "Muchas empresas, rentables en sus inicios, incluso llegaron a desparecer por no saber adaptarse a ese entorno tan cambiante, y tan tirano en tantas ocasiones, que ocasiona que empresas potencialmente exitosas caigan víctimas fulgurantes de una mala, nula o desastrosa implantación de una cultura de innovación” (Martínez, 2006, pág. 23).

La sociedad y la economía del conocimiento que caracterizan a las empresas actualmente suelen conceptualizar la innovación como un factor de diferenciación, pero para la empresa más bien representa un asunto esencial que tiene relación con la supervivencia, ser diferente, operar distinto a cómo lo hace la competencia e inclusive producir bienes o servicios con características 
únicas; esta es una premisa que las organizaciones deberían asumir e incorporar en sus procesos productivos.

Porter (2015) sostiene que para que las empresas logren tener una ventaja competitiva deben realizar acciones estratégicas creativas e innovadoras para ser más eficientes que los competidores, o hacer productos y prestar servicios de manera diferente a la de ellos.

La creatividad e innovación comprenden elementos en los cuales las organizaciones se apoyan para alcanzar el éxito debido a los múltiples beneficios que proporcionan. La creatividad es, por su parte, la capacidad de fabricar o crear algo que resalte del resto por causa de su originalidad o características únicas o exclusivas. La innovación, en cambio, hace referencia a un proceso que permite hacer modificaciones a productos o procesos ya existentes con miras a obtener resultados diferentes. La creatividad y la innovación generan cambio en la industria, pero no se puede limitar la magnitud de los cambios solo a aquellos que sean significativos y de relevancia, puesto que, operando de esta manera, la organización quedaría estancada sin presentar avances de ninguna índole; cualquier cambio, por más insignificante que parezca, en lo posterior puede derivar en futuras innovaciones de importancia para la organización.

La creación de productos nuevos es un proceso interactivo que incluye a todos los miembros del equipo, pero gran parte del éxito o del fracaso en este punto se desprende del liderazgo, capacidad de motivación y estrategia organizacional trazada por la cúpula empresarial.

López, Montes y Vásquez (2007) aseguran que la innovación no es un proceso sencillo pero es necesario; a pesar de ser complejo y arriesgado, no hacer nada no es una opción. En la actualidad, las actividades innovadoras no están reservadas para las grandes empresas: ser una empresa innovadora es cuestión de actitud.

\section{Barreras que pueden obstaculizar la innovación}

La innovación se ha convertido en un factor determinante para la continuidad de las empresas que, conscientes de la exigencia y requerimientos cada vez más altos del mercado, apuestan gran parte de su éxito a productos y servicios con valor agregado para el cliente. Sin embargo, no es raro encontrar organizaciones que siguen repitiendo los mismos productos y utilizando los mismos procesos durante años por temor a implementar cambios que produzcan resultados inciertos o el riesgo que supone la fabricación de un producto que no tiene la garantía de cumplir con las expectativas, requerimientos y preferencias del consumidor.

Gran parte de empresarios está de acuerdo con que es necesario innovar, pero muchas veces ocurre que existe un descontento generalizado por parte de los stakeholders debido a la manera cómo la innovación se pretende implantar en la industria. Es habitual que, cuando se pretenden incluir procesos innovadores dentro de las actividades regulares, haya resistencia por parte de los miembros de la organización, pues cualquier cambio, por más pequeño que sea, generará recelo y temor en el equipo debido a la incertidumbre del resultado final. Por otro lado, existen creencias arraigadas en la mente de muchos directivos empresariales quienes sostienen que la innovación se puede aplicar única y exclusivamente en empresas nuevas y aquellas que tienen años de trayectoria en el mercado no son capaces de implementar procesos productivos diferentes a los que vienen operando durante años. 
Huergo y Jammandreu (2004), referidos por Calvo, Peña, Culebras de Mesa y Gómez (2013) en su libro Estudios sobre innovación tecnológica en España, sostienen que las empresas entrantes presentan alta probabilidad de innovar, capacidad que se atenúa lentamente en el período posentrada, de modo que las empresas con más años en la industria tienden a presentar menores posibilidades de innovar.

Es por esto que contar con líderes que puedan contribuir y garantizar mayor efectividad en la implementación del cambio constituye un factor de desarrollo muy valorado y que todas las organizaciones buscan actualmente. La innovación pretende en el fondo evitar ser mermados lentamente por la competencia. Desafortunadamente, muchas veces son fallidos los intentos por implementar eficientemente procesos innovadores y lo único que se obtiene es un desperdicio de tiempo y recursos. En ocasiones, ocurre que la gerencia está tan pendiente del orden y del trabajo organizado que no comprende con claridad que los tiempos actuales demandan tomarse un tiempo para pensar cómo eliminar la rigidez del trabajo en los stakeholders, cómo reorganizar las actividades o reagrupar a los miembros del equipo con base en sus capacidades y aptitudes; pero, sobre todo, cómo hacer del trabajo algo divertido y sentir pasión por las labores ejecutadas. Esos son cuestionamientos auténticos que todo directivo de empresa debería formularse y pensar en respuestas acertadas que permitan resolver la problemática de las actividades aburridas, monótonas y carentes de sentido para el trabajador.

La innovación tiene un cúmulo de beneficios para las organizaciones, pero equivocadamente se considera que la innovación es una característica de las grandes empresas: la innovación es un término aplicable también a la mediana, pequeña o micro empresa, tal como lo refieren López y otros autores (2007), pues ellos sostienen que la innovación, por paradójico que parezca, se asienta sobre la tradición y, por esta razón, cualquier empresa, pequeña o grande, opere o no en un sector de alta tecnología, puede ser protagonista en ella.

Innovar de forma permanente es necesario; pero, para que esto ocurra, se debe seguir un proceso sistematizado tomando en cuenta que siempre habrá circunstancias que dificulten su ejecución y reduzcan la capacidad innovadora de la industria. "La existencia de barreras a la innovación se hace más intensa cuanto menor es el tamaño de la empresa" (Cerverón e Ybarra, 2016, pág. 29).

Cada empresa está en la necesidad imperiosa de determinar sus propias barreras, ya sean de talento humano, recurso económico, infraestructura o tecnología, y, una vez identificadas, diseñar y ejecutar un plan de acción que permita superar estos obstáculos e implantar procesos innovadores pensados en la obtención de productos o servicios con valor agregado.

\section{La gestión del conocimiento y su aporte al proceso de innovación}

Autores como Nonaka \& Takeuchi (1998) afirman que el conocimiento se entiende como un activo que tiene la capacidad de generar valor y producir riqueza, por lo cual requiere ser protegido para preservar la fuente de la ventaja competitiva de la empresa.

La gestión del conocimiento constituye una herramienta facilitadora del progreso para las organizaciones; sin embargo, cuando se habla de conocimiento, la tendencia común es relacionar sistemas cuya finalidad va direccionada a recibir, sintetizar y distribuir información a manera de 
conocimiento útil para la toma de decisiones en la industria, pero pocas veces se analiza que la gestión del conocimiento sirve además para generar nuevos saberes y, a partir de ellos, incluir la innovación como parte esencial de los procesos productivos en la industria.

Muñoz (2017), en su libro Seis canastas para innovar, afirma que, si bien es cierto la gestión del conocimiento seguirá siendo la clave para tener éxito, debemos entender que los conocimientos pronto quedarán obsoletos y que lo importante es que estos conocimientos se transformen en innovaciones.

Gestionar el conocimiento tiene relación con la formalización de procesos, estrategias e insumos enfocados en el desarrollo y crecimiento del capital intelectual en la empresa; esto persigue la finalidad de utilizar el conocimiento existente en la organización para transformarlo en beneficios tangibles tanto para la industria como para el personal que forma parte de ella. Por su parte, la innovación tiene que ver con la obtención de beneficios valiéndose, para tal efecto, de los recursos que proporciona la gestión del conocimiento. La innovación se ejecuta partiendo de una idea que evoluciona y se va desarrollando, pero, para que exista tal desarrollo y pueda madurar la idea, la comunicación y el trabajo permanente entre los stakeholders son dos elementos importantes que además coadyuvan a la propuesta de ideas diferentes seleccionando aquellas que puedan desplegarse en acciones y transformarse en productos tangibles. Si se juntan ambos conceptos, gestión del conocimiento e innovación, se puede concluir que ambos conceptos persiguen un objetivo en común: la obtención de beneficios para la empresa y también para la sociedad mediante la producción de bienes y servicios mejores, más completos y que satisfagan de mejor manera las exigencias y expectativas del mercado. Por tanto, es mucho más lo que vincula a la gestión del conocimiento con la innovación que lo que las separa.

No existe una estructura formal en las empresas que facilite la utilización del conocimiento en forma efectiva, pero es importante tener en cuenta que los stakeholders se apropian del conocimiento que se genera en la industria con el quehacer de las actividades diarias. El conocimiento, a su vez, se consolida gracias a la interacción constante entre la organización y sus grupos de interés como proveedores, clientes, competidores y entorno empresarial. Es por esto que contar con estrategias que permitan tener acceso oportuno al conocimiento que muchas veces se encuentra presente, pero segregado entre los diferentes departamentos de la organización, es indispensable.

Alavi y Leidner (1999) advierten que la información se convierte en conocimiento una vez que se ha procesado en la mente de un individuo, y el conocimiento se vuelve a convertir en información cuando se articula o comunica a los demás por medio de un texto escrito, en formato electrónico, de forma oral o por otros medios.

La complejidad y multidisciplinariedad del conocimiento latente en las organizaciones presenta barreras y desafíos que limitan su empoderamiento y asimilación por parte de los stakeholders. Por tanto, para convertirlo en una herramienta útil, es necesario clasificarlo y organizarlo de manera que sea accesible para todos los miembros de la empresa y que ellos, a su vez, lo utilicen y contribuyan al proceso de innovación constante en la industria. La apropiación del conocimiento, en la realidad actual de las organizaciones, presenta barreras difíciles de superar en vista de que su dominio y posesión se encuentra entre los diferentes actores que 
dinamizan los procesos en la industria. Esto implica la necesidad de contar en primera instancia con buenos sistemas de fluidez comunicacional, y con políticas y estrategias que faciliten la transmisión del conocimiento entre los diferentes departamentos a fin de que se pueda compartir los saberes generados por cada uno de los miembros de la organización.

"Si el conocimiento no se gestiona de alguna forma, ya sea almacenándolo, o haciéndolo circular, o gestionando las competencia de quienes participan en esa realidad, se corre el riesgo de perder el principal factor diferenciador que promueve la sinergia en la innovación o en el cambio" (Peluffo y Catalán, 2002, pág. 16).

Indudablemente, la capacidad de innovación en la industria se deriva de una sólida base de conocimientos existentes; estos saberes pueden tener diversas tipologías con base en las habilidades, destrezas y experticia de cada miembro de la organización. Es por esto que, para que la innovación se pueda llevar a cabo correctamente, es necesario conocer en todo momento las necesidades del mercado, de otra manera es posible que el producto o servicio no tenga la acogida esperada debido a que no cumple con los requerimientos, gustos y preferencias del consumidor; es decir, la innovación no puede darse al azar o por simple decisión de la gerencia por producir algo diferente, sino que debe ser sistematizada y responder a un estudio previo de las necesidades insatisfechas.

Gupta (2016) señala que la innovación inicia con la identificación de la necesidad por una solución innovadora y termina con la comercialización de la solución innovadora.

\section{La innovación y su importancia en el mundo de los negocios}

Según Corma (2013), los procesos de innovación en la historia de la humanidad han sido un factor que ha hecho posible la creación de una sociedad moderna, industrializada y compleja, y se han constituido como una influyente fuente de poder entre las naciones del mundo.

La forma de hacer negocios ha sufrido cambios importantes hoy en día por causa de factores tales como la globalización, la oferta y la demanda, el poder económico de competidores cada vez más fuertes y la competitividad en un mercado que muestra una saturación importante de opciones para el consumidor. Ante tal situación, se vuelve indispensable la implementación de estrategias que permitan a la industria contar con una ventaja competitiva y puedan comercializar productos o servicios que logren posicionarse con rapidez en el mercado. Actualmente, la decisión de compra del consumidor se ve altamente influenciada por los factores socioeconómicos y culturales del entorno. Por otro lado, muchas organizaciones atienden un mismo nicho de mercado; los clientes demandan nuevos productos o servicios y si la organización no está preparada para ello es claro que de a poco se quedará rezagada y el riesgo de desaparecer con el pasar de los años será mayor. La innovación, por sí sola, no garantiza el éxito empresarial, pero permite a directivos y stakeholders incidir en el crecimiento del negocio y generar cambios importantes en temas de cultura organizacional, sistemas, procesos, productos y servicios, lo cual redunda en el crecimiento personal y profesional del equipo de trabajo.

Morales (2014), en su libro El emprendedor de organizaciones innovadoras, señala que poner en marcha un negocio se constituye en una tarea enormemente ambiciosa. Se necesita el impulso incansable de un equipo de trabajo que debe armar el negocio desde el desarrollo de la 
idea hasta su montaje e implementación interactuando constantemente entre la teoría y la práctica.

Los competidores, que ingresan hoy en día al mercado intentando captar un segmento de potenciales consumidores, se caracterizan por fabricar productos de calidad que satisfagan las expectativas del cliente; sin embargo, del cúmulo de competidores es claro que solo unos cuantos van a perdurar en el tiempo, esto se debe en gran parte no solo a un adecuado plan de mercadeo sino que el modelo de negocio está adaptado y responde a un estudio exhaustivo del mercado objetivo y de la definición de la propuesta de valor.

Según Maqueda (2010), un elemento esencial de la innovación es su aplicación exitosa de forma comercial; no basta solo con inventar un producto o servicio nuevo, lo que genera valor es introducirlo adecuadamente en el mercado y que este lo acepte.

Con el pasar del tiempo, a veces ocurre que muchas innovaciones fallan y eso se debe a que el resultado obtenido por medio de la innovación no responde a un objetivo claro. Ser innovador puede ser tentador para muchas empresas pero, si no se parte de una idea clara del problema que se pretende resolver, es posible que se estén desperdiciando recursos valiosos. En definitiva, las actividades y procesos de innovación, incorporados en la industria, deben estar pensados y ligados a la estrategia y operaciones globales de la empresa. De esta manera, el crecimiento sostenido y perdurable en el tiempo, y la reputación generada entre los consumidores serán los encargados de determinar el éxito o fracaso de las innovaciones realizadas por la organización.

\section{Conclusiones}

La empresa que no innova aumenta el riesgo de desaparecer con el tiempo y volverse irrelevante en el mercado. Las empresas no deben hacerse cuestionamientos si innovar o no hacerlo, puesto que la innovación es una necesidad imperiosa en el entorno actual que envuelve a las organizaciones; por tanto, todos los esfuerzos deben ir enfocados en gestionar la innovación de manera que permita el crecimiento sostenible de las empresas a través de los años.

Cuando las empresas innovan de forma sostenible logran desarrollar una ventaja competitiva que es percibida por el mercado como un plus, como algo único y exclusivo, y esto permite a la organización ocupar un lugar privilegiado en la mente del consumidor. Aquellas que no innovan, tarde o temprano terminarán por desaparecer y serán absorbidas por empresas más grandes. La innovación representa un reto para todas las organizaciones en la actualidad debido al temor e incertidumbre por implementar procesos que no se habían realizado anteriormente. Es natural que, al explorar terrenos desconocidos, el riesgo de equivocarse permanezca latente; pero, con el direccionamiento y estrategia correcta, las probabilidades se inclinarán más en favor del éxito de la innovación que en su fracaso.

El concepto de innovación no se limita a la implementación de ligeras mejoras a los procesos productivos de la industria, abarca en un sentido más amplio todo el proceso de gestión empresarial, de forma tal, que los productos entregados al mercado respondan a una necesidad u oportunidad detectada y que no estaba siendo satisfecha. Durante años se consideró que la 
innovación era una cualidad exclusiva y aplicable solo a las grandes empresas; sin embargo, parte de la naturaleza humana radica en el deseo constante por crear algo nuevo.

Actualmente, la innovación es aplicable a cualquier empresa independientemente de su actividad económica; por tanto, para que este proceso pueda llegar a feliz término, lo único que se necesita es proveer el ambiente y condiciones necesarias para que los stakeholders, de la mano de la gerencia, puedan implementar la innovación como una herramienta estratégica que coadyuve al cambio y al crecimiento empresarial. Muchas veces, las organizaciones toman la decisión de incorporar procesos innovadores como parte esencial de sus actividades regulares, pero desafortunadamente estos intentos por hacer las cosas de manera diferente solo desembocan en un desperdicio de tiempo y recursos económicos.

Esto se genera por causa de un direccionamiento equivocado donde se prioriza la inversión en maquinaria y tecnología por encima de la capacitación a los stakeholders como elemento determinante y eje central en el proceso de innovación. La dirección de un líder que sepa guiar al equipo de trabajo por el sendero que conduce a la consecución de los objetivos empresariales resulta indispensable y necesario para cualquier organización. El contexto actual que envuelve a las organizaciones requiere gerentes con visión y capacidades de motivar, dirigir y desarrollar el talento humano con el que cuenta la empresa.

Las actividades monótonas y rutinarias generan consecuencias negativas para la organización toda vez que los procesos se vuelven obsoletos y, con ello, los productos obtenidos. Es necesario analizar y entender que todo bien o servicio es diseñado e introducido al mercado para satisfacer gustos y preferencias del consumidor en un determinado periodo de tiempo, pero no se puede pretender que, por siempre, un producto tenga la misma acogida y aceptación que tuvo en un inicio; de ahí, se deriva la necesidad de refrescar las ideas y procesos productivos de la industria con un aire de creatividad e innovación. Todo cambio, por pequeño que sea, genera resistencia entre los stakeholders por múltiples razones; es por esto que la innovación debe implantarse en la organización siguiendo un proceso sistematizado, tomando en cuenta los factores que dificultan su ejecución y pueden mermar de alguna manera la capacidad innovadora de la empresa.

\section{Bibliografía}

Alavi, M. y Leidner, D. (1999). Knowledge management systems: issues, challenges, and benefits. Communications of the AIS. 1, (2).

Arboníes, A. (2017). Conocimiento para innovar. Cómo evitar la miopía en la gestión del conocimiento. Madrid: Díaz de Santos, S.A.

Arboníes, A. (2013). La disciplina de la innovación: Rutinas creativas. Madrid: Díaz de Santos, S.A. Bermejo, J., López, M. (2014). La innovación continua en el éxito empresarial. Madrid: UNED.

Calvo, J., Peña, F., Culebras, A., Gómez, A. (2013). Estudios sobre innovación tecnológica en España. Madrid: UNED.

Castillo, A., Cubeiro, J. (2016). Liderazgo Innovador para dummies. Málaga: Planeta de Libros.

Cerverón, V., Ybarra, J. (2016). La innovación empresarial en Ontinyent y su entorno. España: Universidad de Alicante.

Cordero S, P., Chavarría, H., Echeverri, R. y Sepúlveda, S. (2003). Territorios Rurales, Competitividad y Desarrollo. San José: IICA. 
Corma, F. (2013). Innovación, innovadores y empresa innovadora. Madrid: Ediciones Díaz de Santos, S.A.

Domingo, C. (2013). El viaje de la innovación: La guía definitiva para innovar con éxito.

Drucker, P. (2014). La gerencia efectiva. Buenos Aires: Debolsillo.

Dussauge, P. Hart, S. \& Ramantsoa, B. (1992). Strategic Technology Management, Chichester, England: J.Wiley.

García, J. (2009). Gestión de la innovación empresarial: Claves para ser una empresa innovadora. Madrid: NETBIBLO.

Gupta, P. (2016). La innovación como solución. Haciendo la innovación más persuasiva, previsible y rentable. México, DF: Accelper Consulting.

Hamel, Gary. (1998), "Opinion Strategy Innovation and the Quest for Value." Sloan Management Review: 7-14.

López, N., Montes, J., Vásquez, C. (2007). Cómo gestionar la innovación en las pymes. España: Netbiblo, S.L.

Martínez, A., López, P., García, A., Estrada, S. (2009). Innovación y competitividad en la sociedad del conocimiento. México, DF: Plaza y Valdes Editores.

Martínez, L. (2006). Gestión del Cambio y la Innovación en la Empresa. Madrid: Ideaspropias.

Maqueda, J. (2010). Marketing, innovación y nuevos negocios. España: ESIC.

Medellín, E. (2013). Construir la innovación. Gestión de tecnología en la empresa. México, DF: Siglo XXI Editores.

Morales, C. (2014). El emprendedor de organizaciones innovadoras. Bogotá: Siglo del Hombre Editores.

Muñoz, R. (2017). 6 canastas para innovar. El método revolucionario que pondrá la innovación al alcance de todos: México: Penguin Random House.

Muñoz, R. (2014). Innovación a la mexicana: Más allá de romper paradigmas. México DF: Penguin Random House.

Nonaka, I. \& Takeuchi, H. (1998). The knowledge creating company: how Japanese companies create the dynamics of innovation. Oxford, UK: Oxford University Press.

OCDE. (2005). Oslo Manual: Proposed Guidelines for Collecting and Interpreting Innovation Data. Third edition.

OCDE. (2013). Innovación en las empresas. Una perspectiva microeconómica. México DF: Foro Consultivo Científico y Tecnológico, A.C.

Peluffo, M. y Catalán, E. (2002). Introducción a la gestión del conocimiento y su aplicación al sector público. Santiago de Chile: ILPES

Perkins, D. N. (1981). The Mind's Best Work. Cambridge, Mass: Harvard University Press.

Porter, M. (2015). Ventaja Competitiva: Creación y sostenimiento de un desempeño superior. México DF: Grupo Editorial Patria S.A.

Roberts, J. (2006). La empresa moderna. Organización, competencia y resultado. Barcelona: Antony Bosch editor.

Rodríguez, V. (2017). Empresa y Administración. Madrid: Editex.

Rothwell, R. (1994), Industrial Innovation: Success, Strategy, Trends In Dodgson, M.; Rothwell, R., The Handbook of industrial (pp.33-53) USA: Edward Elgar.

Schumpeter, J. (1967). Teoría del desenvolvimiento económico. México D.F: Fondo de Cultura Económica.

Valdivia, J. (2015). Comercialización de productos y servicios en pequeños negocios o microempresas. Madrid: IC Editorial

Verde, J. (2013). Soñar es poder. Barcelona: Penguin Random House Grupo Editorial. 